\title{
Functional Changes in the Care-needing Elderly after Surface Electrical Stimulation to the Abdomen
}

\author{
Misa MiURA ${ }^{1}$, Kazunori SEKI ${ }^{2}$, Osamu ITO ${ }^{1}$, Yasunobu HANDA ${ }^{3}$ and Masahiro KOHZUKI ${ }^{1}$ \\ 1) Department of Internal Medicine and Rehabilitation, Tohoku University Graduate School of Medicine, Sendai, Japan \\ 2) Sendai School of Health and Welfare, Sendai, Japan \\ 3) Division of Developmental Neuroscience, Tohoku University Graduate School of Medicine, Sendai, Japan
}

\begin{abstract}
Background: Strength of the trunk muscles is a key component of motor control, but it declines easily with the process of aging and/or disuse. Objectives: To investigate the effects of surface electrical stimulation (ES) to the abdominal muscles and the motor performance for care-needing elderly. Design: Controlled trial. Participants: Twenty-one elderly people (60-90 years) with care-needing, who were admitted to a nursing home or hospital for daily care and their score of Barthel Index had been stable from $55.3 \pm 19.4$ to $55.3 \pm 19.8$ during 3 months before intervention. Intervention: Common physical/occupational therapy was performed for all of the subjects during the study period. ES was applied to the abdomen of 15 subjects (ES group) twice a day for 8 weeks. Measurements: Functional measurements were performed before and after beginning of the study with some parameters as follows: grip strength; movement time for sitting up (MSU); maximum walking speed (WS). Results: No significant differences between two groups were noted for characteristics at baseline. In the ES group, MSU (from $21.1 \pm 21.4$ to $10.4 \pm 8.3 \mathrm{sec}$ ) significantly improved $(\mathrm{p}<0.05)$ after 8weeks. WS (from $17.7 \pm 12.1$ to 24.3 $\pm 15.3 \mathrm{~m} / \mathrm{min}$ ) were also improved $(p<0.05)$ after 8 weeks. Limitations: A limitation of this study was the small sample size. Conclusions: ES to the abdominal area has the potential to improve motor function in the care-needing elderly through reinforcement of the abdominal muscles.
\end{abstract}

Key words: abdominal muscles, care-needing elderly, surface electrical stimulation

(J Jpn Phys Ther Assoc 15: 15-20, 2012)

In recent years, with the increasing number of elderly people in Japan, preventive care ${ }^{1)}$ has become of considerable importance. Loss of muscle strength with aging has been suggested to be one of the main factors causing functional limitations in the activities of daily living, such as rising from a chair or climbing up and down stairs ${ }^{2-4}$. The strength of the trunk muscles declines easily with the process of aging and/or disuse, and these muscles have important roles not only for trunk function but also for the whole body motor function ${ }^{5)}$. The subscore of the functional independence measures relating to locomotion-transfers in stroke subjects at discharge was reported to be positively correlated with trunk muscle torque values, except for iso-

Received: May 28, 2012

Accepted: September 13, 2012

Advance Publication by J-STAGE: October 26, 2012

Correspondence to: Misa Miura, Department of Internal Medicine and Rehabilitation, Tohoku University Graduate School of Medicine, 1-1 Seiryo-machi, Aoba-ku, Sendai 980-8574, Japan.

e-mail:m.miura@med.tohoku.ac.jp metric extension ${ }^{6}$.

Machine training, aerobics and other activities are recommended for health promotion in the elderly, but there are few programs available for bedridden elderly patients those who deteriorated of the will to strengthen their weak abdominal muscles. Some methods of physical therapy, including electrical stimulation (ES), have been used for muscle strengthening. In particular, ES has been adopted to improve not only muscle atrophy but also impaired motor function in hemiplegic patients and spinal cord-injured patients since the 1980s, and its safety and effectiveness have been confirmed ${ }^{7-11)}$. In the present study, we applied surface ES to the abdominal muscles of care-needing elderly people and investigated its effects in motor performance.

\section{Methods}

Subjects and Overall Design

This prospective randomized study was carried out in 
Table 1. Demographic and clinical parameters of the subjects

\begin{tabular}{cccc}
\hline & $\begin{array}{c}\text { ES group } \\
(\mathrm{n}=15)\end{array}$ & $\begin{array}{c}\text { Ctrl group } \\
(\mathrm{n}=6)\end{array}$ & $\mathrm{P}$ \\
\hline & $77.9 \pm 8.9$ & $73.5 \pm 8.2$ & 0.19 \\
Height $(\mathrm{cm})$ & $150.2 \pm 9.9$ & $152.3 \pm 12.4$ & 0.97 \\
Weight $(\mathrm{kg})$ & $54.9 \pm 12.3$ & $48.6 \pm 11.1$ & 0.17 \\
BMI & $24.1 \pm 4.1$ & $21.0 \pm 3.9$ & 0.17 \\
\hline
\end{tabular}

Values are presented as the mean $\pm \mathrm{SD}$. No significant differences beween two groups were noted for characteristics at baseline. BMI: body mass index.

21 elderly people (10 males and 11 females) who were admitted to an institute or a hospital for nursing care. The subjects were prolonged decrease in muscle contractile activity, such as that in a sedentary lifestyle, during periods of prolonged bed rest, inactivity as a consequence of congestive heart failure, stroke, COPD, limb casting and muscle unloading (i.e., microgravity). Although continuous intervention with common physical therapy was performed, their score of Barthel Index had been stable from $55.3 \pm 19.4$ to $55.3 \pm 19.8$ during 3 months before the start of the study owing to chronic disuse and they could walk with using the device (cane, walker, orhosis and so on) They had spent the major part of each day in their bedroom. The subjects were divided into two groups. Before study started, the subjects were fully informed about the protocol, and provided informed consent to participate in the study. The study protocol was approved by the Institutional Review Board of Tohoku University Graduate School of Medicine including the ethical rules for human experimentation that are stated in the Declaration of Helsinki.

Participants were randomly assigned to 2 groups. ES to the abdominal muscles was performed in one group in addition to the physical/occupational program (ES group; nine males and six females $n=15$ ), while the physical/occupational program that depends on personal physical ability, such as ROM ex, muscle-strength ex and so on, alone was performed in the other group as a control (Ctrl group; four males and two females $n=6$ ). Randomization was carried out electrically by a physical therapist who was not informed about this study. The demographic and clinical parameters of the subjects at the beginning of the study are summarized in (Table 1). There were no differences between the two groups for any of the items. All of the subjects underwent a standard physical/occupational therapy program twice a week during the study period.

\section{Procedures}

ES was applied to the abdominal muscles including the obliquus externus abdominis muscle (EO), obliquus internus abdominis muscle (IO), transversus abdominis muscle ( $\mathrm{Tr}$ ) and rectus abdominis muscle (Rc) on both sides. Surface electrodes of $5 \times 10 \mathrm{~cm}$ (Lintec Co., Tokyo, Japan)

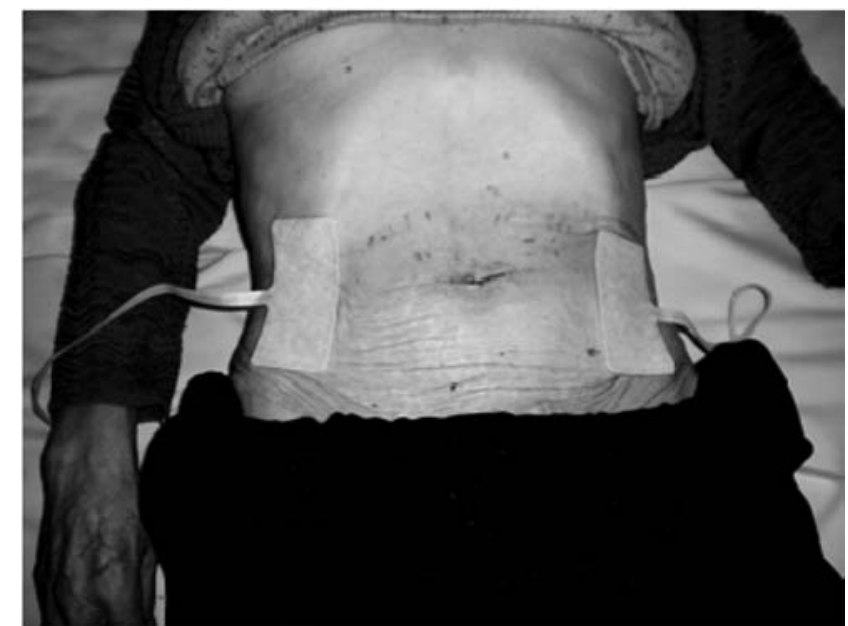

Fig. 1. Positions of the electrodes. The stimulation electrodes were placed on the trunk over the abdominal oblique muscles.

were placed on the skin covering the muscles, except for the Rc, which was located below the inferior border of the 12th rib. A portable electrical stimulator (Nodoka; Lintec Co.) was used for ES. Cyclic stimulation with 10 sec ON and 5 sec OFF was performed for 15 min twice a day for 8 weeks (Fig. 1), separately from the physical/occupational therapy. Bidirectional rectangular pulses with 0.2 msec duration were used and frequency of the pulse train was $30 \mathrm{~Hz}$ in each direction. Thus we confirmed that EO, IO, Tr and Rc were stimulated by this method to a normal adult before the study start. The stimulation intensity was set at the maximum level just below the pain threshold so that the subjects never perceived any unpleasant feelings. At the beginning of the study and after 8 weeks (8W), performance tests were carried out for all of the subjects to evaluate the changes in their physical functions.

\section{Performance Tests}

Movement time required for sitting up (MSU)

Rising from lying supine on a physiotherapy bench to sitting on the edge and rising to sitting were measured ${ }^{12}$. Two trials were performed successively and the fastest time 
Table 2. Changes in the Parameters of Motor Performance

\begin{tabular}{ccccccc}
\hline & \multicolumn{3}{c}{$\begin{array}{c}\text { ES group } \\
(\mathrm{n}=15)\end{array}$} & & & \multicolumn{3}{c}{$\begin{array}{c}\text { Ctrl group } \\
(\mathrm{n}=6)\end{array}$} \\
\cline { 2 - 7 } & before & After 8W & $\mathrm{P}$ & before & After 8W & $\mathrm{P}$ \\
\hline MSU(s) & $21.1 \pm 21.4$ & $10.4 \pm 8.3^{*}$ & 0.029 & $18.0 \pm 17.3$ & $17.1 \pm 17.2$ & 0.74 \\
WS(m/min) & $17.7 \pm 24.3$ & $24.3 \pm 15.3^{*}$ & 0.017 & $9.3 \pm 10.3$ & $7.2 \pm 7.7$ & 0.77 \\
grip (kg) & $19.4 \pm 9.7$ & $21.1 \pm 10.7$ & 0.20 & $11.2 \pm 5.5$ & $12.1 \pm 5.4$ & 0.10 \\
\hline
\end{tabular}

Values are presented as the mean \pm SD. No significant differences between two groups were noted for characteristics at baseline. ${ }^{*} P<0.05$, significant difference from the value at the beginning of the study. MSU: Movement time required for sitting up WS: Walking speed for $10 \mathrm{~m}$ distance with maximum effort

was used for the calculations. The bench used was a semifirm bench with a plastic-covered surface, $200 \times 100 \mathrm{~cm}$ with a height of $55 \mathrm{~cm}^{12)}$.

\section{Grip strengths}

For grip strength measurements, a digital hand dynamometer (Model T.K.K.5401, Takei Kogyou, Inc., Tokyo) was used. The test was administered by a trained physical or occupational therapist and highest of two measures in the dominant hand was used in the analysis ${ }^{13)}$.

Walking speed for $10 \mathrm{~m}$ distance with maximum effort (WS)

The patient began to walk as quickly as possible $3 \mathrm{~m}$ before the starting line, and the timing commenced when the patient's swing leg crossed the starting line and ended when the patient's swing leg passed over the finish line by physical therapists or occupational therapists that were unaware of the information of the groups. Two trials were performed successively and the fastest time was used for the calculations ${ }^{14)}$.

\section{Measurement of the Cross-sectional Area}

We examined the morphologic changes of the abdominal muscles using Computed Tomography (CT) in only one subject by a Radiological technologist. The cross-sectional area of the abdominal muscles was measured at the level of the umbilicus. The level of the middle of vertebra L3 was located using the CT scout image (Althen 2005) as a guide, and eFilm software (Image J; New York) employed to estimate the area using an ellipse draw and analysis tool. The results generated by the draw and analysis tool are indicated on the image. The cross-sectional areas of the Rc and flank muscles including the EO, IO and Tr were calculated on each side by drawing the external margin of each muscle group. These methods were described previously ${ }^{15)}$.

\section{Statistical Analysis}

All data are presented as means \pm SD. Statistical analysis was performed using Shapiro-wilk test and Levene test, then the paired Student t test to compare within-group values before and after the intervention and the unpaired Stu- dent $t$ test was used to compare unpaired data between both groups, and using Stat Cel QC for Windows. Values of $P<$ 0.05 were considered to indicate statistical significance.

\section{Results}

\section{Changes in the Functional Parameters}

No significant differences between two groups were noted for characteristics at baseline. In the ES group, MSU was significantly decreased after $8 \mathrm{~W}(P<0.05)$, while nonsignificant change was observed in the Ctrl group. (Table 2). Compared with the initial value, WS was significantly increased $(P<0.05)$ in the ES group, while non-significant change was found in the Ctrl group during the study (Table 2).

The grip strength which indicates whole body function showed non-significant changes in both groups.

Changes in the cross sectional area of abdominal muscles

Though abdominal CT was examined by a Radiological technologist only in one subject, the increase of abdominal muscle volume was confirmed in this case (Fig. 2). The increase of abdominal muscle volume from $952 \mathrm{~mm}^{2}$ to $1329 \mathrm{~mm}^{2}$ in this case could indicate the local effects of ES.

\section{Discussion}

The aim of this study was to examine the effects of abdominal surface ES on the functions of a limited part of the body and the whole body. We evaluated the effects of ES to the abdomen on selected functional parameters in care-needing elderly subjects. In summary, ES to the abdomen may be able to change care-needing elderly people to active.

In this study, we confirmed the improvement of several functional parameters such as the MSU and WS in the ES group. The improvements were confirmed after 8W. Although the background mechanism underlying such functional changes will be complicated, certain factors can be indicated. 


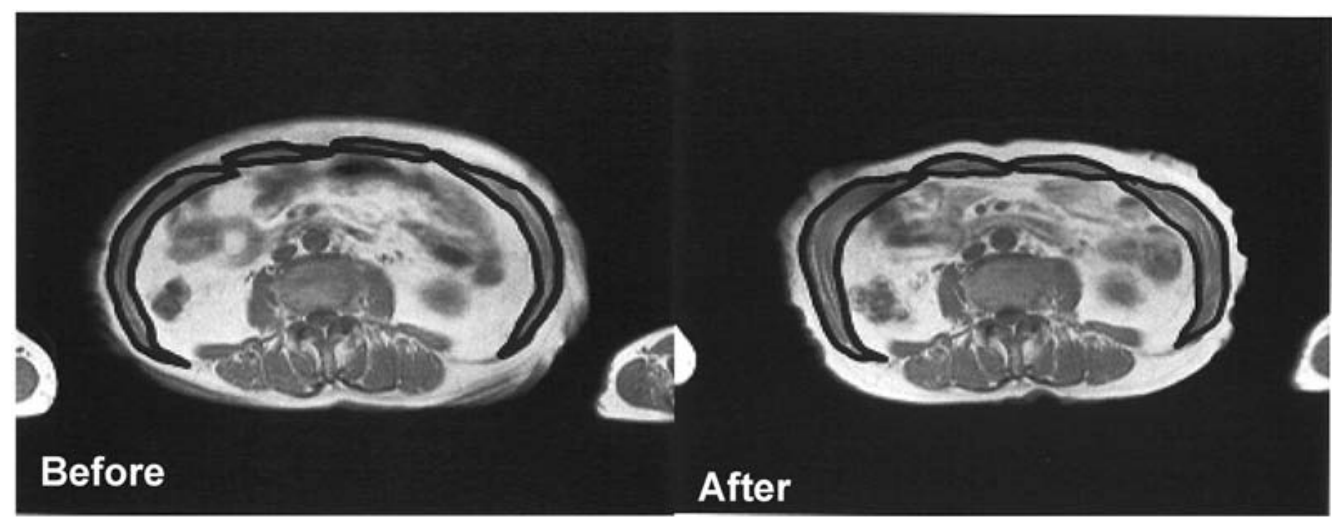

Fig. 2. A change in the abdominal CT was examined only in one subject by Computed tomography. The increase of abdominal muscle volume from $952 \mathrm{~mm}^{2}$ to $1329 \mathrm{~mm}^{2}$ in this case could indicate the local effects of ES.

First, morphologic changes such as (Fig. 2) to the abdominal muscles may provide increased muscle strength. There are several reports showing that muscle strength improves together with an increase in the cross-sectional area in skeletal muscles ${ }^{16-18)}$. Accordingly, it is possible that the ES group obtained more strength of the abdominal muscles than the Ctrl group and the improvements in the MSU in the ES group may have been provided by such increases in the muscle strength.

Second, physiologic changes in the abdominal muscles may be involved in an increase in muscle strength. This finding suggests that the number of neuromuscular units in the abdominal muscles was increased after ES. In other words, the strength of the abdominal muscles may have been improved by an increase in the number of neuromuscular units.

In general, increased muscle strength is provided by changes in neural factors and hypertrophy of the muscle fibers. Although it was recently reported that muscle strength is often brought about by hypertrophy through exercise $^{19)}$, an increase in muscle strength during the early period of training can be caused by an increase in the number of muscle fibers participating in the muscle contraction $^{20)}$. According to Moritani and de Vries, ${ }^{21)}$ both young and elderly groups showed an increase in muscle strength as a result of muscle-strengthening exercises for $8 \mathrm{~W}$, but muscle hypertrophy was only observed in the young group. Consequently, the authors concluded that the increase in muscle strength in the elderly group after exercise occurred through an increase in the number of neuromuscular units rather than muscle hypertrophy. In the present study, we found improvement of the MSU in the ES group. Eriksson et al. ${ }^{22)}$ reported that ES required $8 \mathrm{~W}$ to bring about muscle hypertrophy. Therefore, it is suspected that the early improvement of motor function after intervention with ES was caused by physiologic changes in the abdominal muscles, similar to the case for physical exercise.
The WS was significantly changed after $8 \mathrm{~W}$ in the ES group, but not in the Ctrl group. From the viewpoint of normal development, standing and walking can be realized on the basis of motions on a bed, such as sitting up. Therefore, it is valid to consider that the late improvements in the WS followed the early improvements in the MSU, reflecting motions on a bed. The increase in the abdominal muscle strength obtained after the initiation of ES may contribute to subsequent facilitation of standing and walking. On the other hand, the grip strengths showed no changes during the study period. Grip strength is an indicator of muscle strength in the whole body. The findings for the grip strengths suggested that global changes relating to the whole body function did not occur in the present study. Therefore, the effects of ES to the abdomen are limited to the local functions executed by the abdominal muscles.

The trunk muscles have important roles not only for trunk function but also for whole body motor function, and contribute to breathing, utterance and defecation. However, the strength of these muscles declines easily with the process of aging. It has been reported that muscle atrophy is based on the aging characteristics of type II fibers ${ }^{23-25)}$ and decreased acceleration of muscle power ${ }^{26)}$. Therefore, reinforcement of type II fibers rather than type I fibers is required for the elderly ${ }^{27)}$. However, effective rehabilitation programs for care-needing elderly people with limited mobility, who spend most of their time on a bed, are limited to exercises involving low loads or stretching, and therefore positive effects on muscle strength cannot be expected with such programs. Several investigators have examined the responses of muscle strength to repeated sessions of ES, and reported increased strength of the skeletal muscles, improved muscle contraction in patients after knee surgery and reduced atrophy ${ }^{22,28-30)}$. These findings indicate that ES can prevent and improve disuse owing to immobilization. ES also produces immediate changes in the motor units, by increasing the recruitment number and action potential ve- 
locity and establishing the selective recruitment of type II fibers $^{31)}$. The safety and efficacy of ES to the skeletal muscles have recently been confirmed, even for heart failure patients $^{32}$. Therefore, ES to the abdominal area has good potential for improving the motor function of care-needing elderly people through abdominal muscle strengthening.

Some potential limitations of our study should be considered. First, this study involved a relatively small number of subjects. Second, we did not measure muscle activities and cross sectional area of the abdominal muscles. Futures studies with more than thirty numbers of subjects (using $\mathrm{G}^{*}$ power for Windows) and muscle activities and cross sectional area of abdomen are necessary to clarify the precise and long-term effects of ES to the abdomen.

\section{Conclusions}

This study is the first controlled trial aiming to show the effectiveness of surface ES to the abdomen for careneeding elderly people. The present results revealed functional improvements in the ES group. We conclude that the results of this study are unique and that surface ES to the abdomen should be taken into consideration as one of the rehabilitation programs for the care-needing elderly.

\section{References}

1) Ishibashi T, Ikagami N: Should the provision of home help services be contained?: Validation of the new preventive care policy in japan. BMC Health Serv Res. 2010, 10:224.

2) Odding E, Valkenburg H, Grobbee D, Hofman A, Pols H: [locomotor disability in the elderly; the ergo study (erasmus rotterdam health and the elderly). Ergo study group]. Ned Tijdschr Geneeskd. 1995, 139: 2096-2100.

3) Guralnik J, Ferrucci L, Simonsick E, Salive M, Wallace R: Lower-extremity function in persons over the age of 70 years as a predictor of subsequent disability. N Engl J Med. 1995, 332: 556-561.

4) Schenkman M, Hughes M, Samsa G, Studenski S: The relative importance of strength and balance in chair rise by functionally impaired older individuals. J Am Geriatr Soc. 1996, 44: 1441-1446.

5) Buchman AS, Boyle PA, Leurgans SE, Evans DA, Bennett DA: Pulmonary function, muscle strength, and incident mobility disability in elders. Proc Am Thorac Soc. 2009, 6: 581587.

6) Karatas M, Cetin N, Bayramoglu M, Dilek A: Trunk muscle strength in relation to balance and functional disability in unihemispheric stroke patients. Am J Phys Med Rehabil. 2004, 83: 81-87.

7) Gordon T, Mao J: Muscle atrophy and procedures for training after spinal cord injury. Phys Ther. 1994, 74: 50-60.

8) Winslow J, Jacobs P, Tepavac D: Fatigue compensation during fes using surface emg. J Electromyogr Kinesiol. 2003, 13: $555-568$.

9) Hillegass E, Dudley G: Surface electrical stimulation of skeletal muscle after spinal cord injury. Spinal Cord. 1999, 37:
251-257.

10) Ochi M, Tabara Y, Kido T, Uetani E, Ochi N, Igase M, Miki $\mathrm{T}$, Kohara K: Quadriceps sarcopenia and visceral obesity are risk factors for postural instability in the middle-aged to elderly population. Geriatr Gerontol Int. 2010, 10: 233-243.

11) Coghlan S, Crowe L, McCarthyPersson U, Minogue C, Caulfield B: Electrical muscle stimulation for deep stabilizing muscles in abdominal wall. Conf Proc IEEE Eng Med Biol Soc. 2008, 2008: 2756-2759.

12) Bramell-Risberg E, Jarnlo GB, Minthon L, Elmstahl S: Lower gait speed in older women with dementia compared with controls. Dement Geriatr Cogn Disord. 2005, 20: 298-305.

13) Giampaoli S, Ferrucci L, Cecchi F, Lo Noce C, Poce A, Dima F, Santaquilani A, Vescio MF, Menotti A: Hand-grip strength predicts incident disability in non-disabled older men. Age Ageing. 1999, 28: 283-288.

14) Suzuki K, Imada G, Iwaya T, Handa T, Kurogo H: Determinants and predictors of the maximum walking speed during computer-assisted gait training in hemiparetic stroke patients. Arch Phys Med Rehabil. 1999, 80: 179-182.

15) Ng J, Kippers V, Richardson C: Muscle fibre orientation of abdominal muscles and suggested surface emg electrode positions. Electromyogr Clin Neurophysiol. 1998, 38: 51-58.

16) Ackermann RT, Marrero DG, Hicks KA, Hoerger TJ, Sorensen S, Zhang P, Engelgau MM, Ratner RE, Herman WH: An evaluation of cost sharing to finance a diet and physical activity intervention to prevent diabetes. Diabetes Care. 2006, 29 : 1237-1241.

17) Dal Corso S, Nápolis L, Malaguti C, Gimenes A, Albuquerque A, Nogueira C, De Fuccio M, Pereira R, Bulle A, McFarlane N, Nery L, Neder J: Skeletal muscle structure and function in response to electrical stimulation in moderately impaired copd patients. Respir Med. 2007, 101: 1236-1243.

18) Verdijk L, Gleeson B, Jonkers R, Meijer K, Savelberg H, Dendale P, van Loon L: Skeletal muscle hypertrophy following resistance training is accompanied by a fiber type-specific increase in satellite cell content in elderly men. J Gerontol A Biol Sci Med Sci. 2009, 64: 332-339.

19) Folland J, Williams A: The adaptations to strength training: Morphological and neurological contributions to increased strength. Sports Med. 2007, 37: 145-168.

20) Cormie P, McCaulley G, McBride J: Power versus strengthpower jump squat training: Influence on the load-power relationship. Med Sci Sports Exerc. 2007, 39: 996-1003.

21) Moritani $T$, deVries $H$ : Potential for gross muscle hypertrophy in older men. J Gerontol. 1980, 35: 672-682.

22) Eriksson E, Häggmark T: Comparison of isometric muscle training and electrical stimulation supplementing isometric muscle training in the recovery after major knee ligament surgery. A preliminary report. Am J Sports Med. 1979, 7: 169-171.

23) Kararizou E, Manta P, Kalfakis N, Vassilopoulos D: Age-related morphometric characteristics of human skeletal muscle in male subjects. Pol J Pathol. 2009, 60: 186-188.

24) Lexell J, Taylor C, Sjöström M: What is the cause of the ageing atrophy? Total number, size and proportion of different fiber types studied in whole vastus lateralis muscle from 15to 83-year-old men. J Neurol Sci. 1988, 84: 275-294. 
25) Porter M, Vandervoort A, Lexell J: Aging of human muscle: Structure, function and adaptability. Scand J Med Sci Sports. 1995, 5: 129-142.

26) Saini A, Faulkner S, Al-Shanti N, Stewart C: Powerful signals for weak muscles. Ageing Res Rev. 2009, 8: 251-267.

27) Takano $Y$, Haneda $Y$, Maeda T, Sakai $Y$, Matsuse H, Kawaguchi T, Tagawa Y, Shiba N: Increasing muscle strength and mass of thigh in elderly people with the hybrid-training method of electrical stimulation and volitional contraction. Tohoku J Exp Med. 2010, 221: 77-85.

28) Kondrashin N, Kots I, Nikulin I, Delov V, Novikov I: [electrostimulation training of the muscles in the stumps of truncated extremities]. Ortop Travmatol Protez. 1975, 56-60.

29) Romero J, Sanford T, Schroeder R, Fahey T: The effects of electrical stimulation of normal quadriceps on strength and girth. Med Sci Sports Exerc. 1982, 14: 194-197.

30) Currier D, Mann R: Muscular strength development by electrical stimulation in healthy individuals. Phys Ther. 1983, 63: 915-921.

31) Montes Molina R, Tabernero Galan A, Martin Garcia M: Spectral electromyographic changes during a muscular strengthening training based on electrical stimulation. Electromyogr Clin Neurophysiol. 1997, 37: 287-295.

32) Dobsak P, Novakova M, Fiser B, Siegelova J, Balcarkova P, Spinarova L, Vitovec J, Minami N, Nagasaka M, Kohzuki M, Yambe T, Imachi K, Nitta SI, Eicher JC, Wolf JE: Electrical stimulation of skeletal muscles - an alternative to aerobic exercise training in patients with chronic heart failure? International Heart Journal. 2006, 47: 441-453. 